\title{
A Nonlinear System Control Strategy Based on Single Chip Computer
}

\author{
Chunmei $\mathrm{Li}^{1, \mathrm{a}}$ * \\ ${ }^{1}$ School of Electrical Engineering, University of Jinan, Shandong, Jinan City, 250022, China \\ ${ }^{a}$ cse_lcm@ujn.edu.cn
}

Keywords: Nonlinear system control strategy, Nonlinear process parameter correction, Liquid level process, nonlinear models.

Abstract. A nonlinear model of a liquid level process is obtained on the analysis of its nonlinear characteristics, finding the basis of its nonlinear process parameter correction and a nonlinear system control strategy based on single chip computer is presented. By measuring the liquid level and flow rate, two nonlinear process parameters of the liquid level process, the amplification coefficient and time constant under different load are calculated according to the basis of the nonlinear process parameter correction. Then the control signal is calculated and the system is run according to the nonlinear system control strategy. A test results is given and it shows that the nonlinear system control strategy is better then the linear system. The maximum overshoot is reduced by $16 \%$.

\section{Introduction}

We know that the most industrial processes are nonlinear. Their process parameters, such as amplification coefficient $K$ and time constant $T$, are variable with the change of the process load. That brings a lot of trouble when we apply the linear principles of automatic control to nonlinear industrial process. For better control effect we must correct the control parameters PID with the change of $K$ and $T$. The key to solve this problem is how to find the scientific basis of the nonlinear process parameter correction. It is solved well by a nonlinear system control strategy based on single chip computer presented in this paper. The test result shows that it improves the control quality considerably.

\section{The Linear and Nonlinear Models of the Liquid Level Process}

The Linear Model of the Liquid Level Process. Fig.1a shows a liquid level process, the inflow is $Q_{\mathrm{i}}$, the outflow is $Q_{\mathrm{o}}$, the fluid is water, when process is stable, $Q_{\mathrm{i}}=Q_{\mathrm{o}}$, the controlled variable liquid level $h_{\mathrm{o}}$ remain unchanged. When the control valve suddenly open a few bigger, the valve core move $\Delta x$, the $Q_{\mathrm{i}}$ has a step input, then $Q_{\mathrm{i}}>Q_{\mathrm{o}}$, Let the load valve unchanged, the $h$ will rise gradually. With the $h$ increasing the $Q_{\mathrm{o}}$ increase gradually and $Q_{\mathrm{i}}=Q_{\mathrm{o}}$ again in the end. The process reached a new balance. Its step response curve shows in Fig. $1 \mathrm{~b}$. In this process let the difference of the $Q_{\mathrm{i}}$ and $Q_{\mathrm{o}}$ is $\Delta Q$, there is

$$
\Delta Q=\Delta Q_{\mathrm{i}}-\Delta Q_{\mathrm{o}}=\frac{d V}{d t}=\mathrm{A} \frac{d \Delta h}{d t}
$$

Where, $\Delta Q_{\mathrm{i}}, \Delta Q_{\mathrm{o}}$ - the micro variables of the $Q_{\mathrm{i}}$ and $Q_{\mathrm{o}}$;

$d V$ - the micro variable of liquid in the cistern;

A — the cross-sectional area of the cistern, here is a constant;

$\Delta h$ - the micro variable of $h_{\mathrm{o}}$.

The micro variable of the $Q_{\mathrm{i}}$ is related with control valve opening $\Delta x$, that is

$\Delta Q_{\mathrm{i}}=k_{\mathrm{x}} \Delta x$

Where, $k_{\mathrm{x}}$ - the flow coefficient of the control valves, known.

Keep the $h_{\mathrm{o}}$ above a certain height, the fluid in load valve will be in turbulent flow condition, the relation between the $Q_{\mathrm{o}}$ and $h_{\mathrm{o}}$ is

$Q_{\mathrm{o}}=k \sqrt{h_{\mathrm{o}}}$

Where, $k$ the flow coefficient of the load valve, it is a constant in turbulent flow condition. 


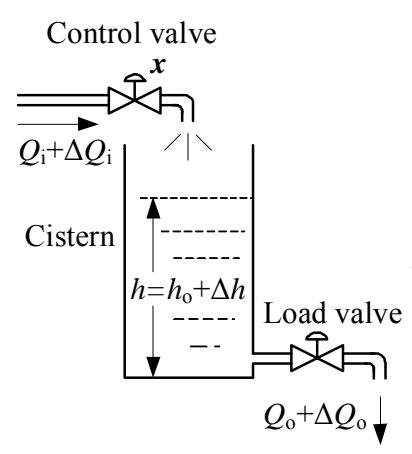

a

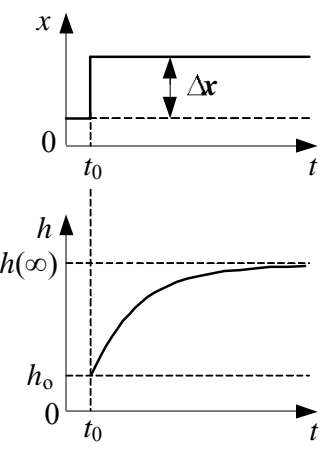

b

Fig. 1 The liquid level process and step response curve

Formula (3) shows it is a nonlinear relation between the $Q_{\mathrm{o}}$ and $h_{\mathrm{o}}$. Therefore, the model of the liquid level process will also be nonlinear. Usually, we make the process linearization and get the micro variable $\Delta Q_{\mathrm{o}}$ as

$$
\begin{aligned}
& \Delta Q_{\mathrm{o}}=\frac{k \Delta h}{2 \sqrt{h_{\mathrm{o}}}} \\
& \text { Let } \frac{k}{2 \sqrt{h_{\mathrm{o}}}}=\frac{1}{R}
\end{aligned}
$$

Where, $R \longrightarrow$ The flow resistance.

Then $\Delta Q_{\mathrm{o}}=\frac{1}{R} \Delta h$ is

Put the formula (6) and (2) into (1), the time domain mathematical model of the liquid level process

$$
R \mathrm{~A} \frac{d \Delta h}{d t}+\Delta h=k_{\mathrm{x}} R \Delta \mathrm{x}
$$

Change to the general form as

$R C \frac{d \Delta h}{d t}+\Delta h=K \Delta x$

or $\quad T \frac{d \Delta h}{d t}+\Delta h=K \Delta \mathrm{x}$

Where, $C$ - Liquid capacity, here $C=\mathrm{A}$;

$T$ - the time constant of the liquid level process;

$K$ - the amplification coefficient of the liquid level process.

and

$T=R C$

$K=k_{\mathrm{x}} R$

So the transfer function of the liquid level process as linear system is

$$
\frac{H(s)}{X(s)}=\frac{K}{T s+1}
$$

Formula (12) is the linear model of the liquid level process showed in Fig.1. It is the result of linearization. When making a liquid level control system according to formula (12), we consider the $K$ and $T$ as a constant. This is the basic method when we use the linear principles of automatic control to run nonlinear control system. But in fact the $K$ and $T$ are not constant. From formula (5) we know that the $R$ is not a constant, it is nonlinear quadratic function relation with the $h_{\mathrm{o}}$. From formula (10) and (11) we can know that the $K$ and $T$ are changed with $h_{\mathrm{o}}$ too. When $h_{\mathrm{o}}$ changed enough value, the $K$ and $T$ have a big change. This will be certain effect to the control accuracy.

The Nonlinear Model of the Liquid Level Process. Now let's solve the problem above. According to formula (5), we can get the $R$ as 
$R=\frac{2 \sqrt{h_{\mathrm{o}}}}{k}$

put formula (10), (11) and (13) into (12), we can built the nonlinear model as

$\frac{H(s)}{X(s)}=\frac{K}{T s+1}=\frac{k_{\mathrm{x}} R}{R C s+1}=\frac{k_{\mathrm{x}} \frac{2 \sqrt{h_{\mathrm{o}}}}{k}}{\frac{2 \sqrt{h_{\mathrm{o}}}}{k} C s+1}$

Where

$K=k_{\mathrm{x}} \frac{2 \sqrt{h_{\mathrm{o}}}}{k}$

$T=\frac{2 \sqrt{h_{\mathrm{o}}}}{k} C$

The $k$ is flow coefficient of the load valve and depending on the load valve opening. We can get it through an experiment. The $k_{\mathrm{x}}$ is the flow coefficient of the control valves and is known. So, the $K$ and $T$ in formula (15) and (16) contain the information of $h_{\mathrm{o}}$. As $h_{\mathrm{o}}$ changes, $K$ and $T$ change. They can be calculated according to the formula (15) and (16) in real-time. This is the nonlinear process parameter correction. Now, so long as we calculate the control signal with the corrected $K$ and $T$, the optimal control in the different $h_{\mathrm{o}}$ can be achieved. That can make the control precision have a lot of improvement. Formula (14) is the nonlinear model of the liquid level process shown in Fig.1, it is the basis for the automatic control of nonlinear system and the modeling is very simple.

\section{The Nonlinear System Control Strategies}

For the nonlinear liquid level process shown in the Fig.1a, installing a liquid level measuring instrument, measuring the $h$, calculating the $K$ and $T$ according to formula (15) and (16) separately and the parameters identification of the nonlinear model formula (14) is finished. Then the control signal is calculated by single chip computer signal acquisition and PI operation control unit according to the selected control scheme. The nonlinear system automatic control of the liquid level process can be realized. Such a nonlinear control system is showed in Fig.2. When using single chip computer, it is very simple and easy to realize such nonlinear system control strategy.

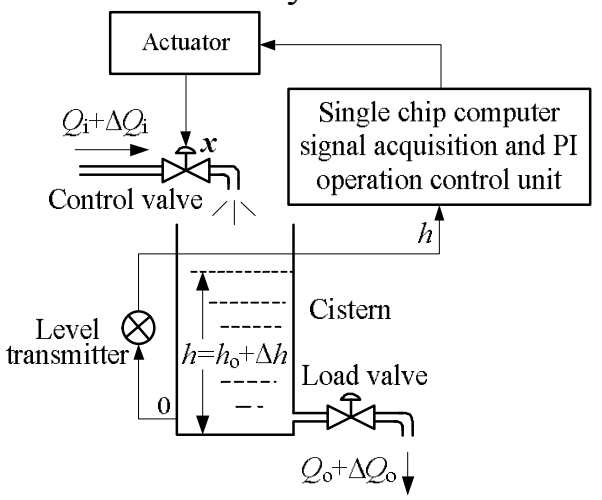

Fig.2 The nonlinear control system of the liquid level process based on $h$

When we put the liquid level process as a linear system, the control signal is calculated according to the error of measured value and set value. The $K$ and $T$ don't need correction. This is the basic principle of linear control system. When we put the liquid level process as a nonlinear system, in addition to calculating the control signal, the $K$ and $T$ do need correction. This is the difference between the linear system and nonlinear system. How to find the basis of the nonlinear process parameter correction is the foundation of realizing nonlinear control. We can get this basis through the theoretical analysis, experiment and experience generally. This is the key for the automatic control of the nonlinear process. The nonlinear control system block diagram is showed in Fig.3. The signal of the nonlinear process parameter correction is marked in Fig.3. 


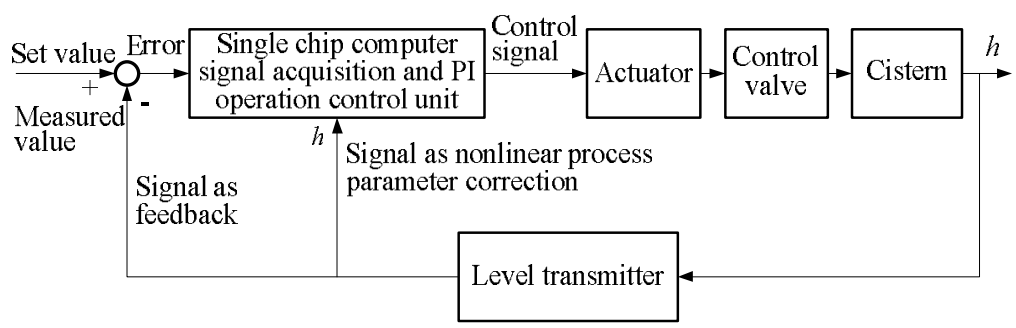

Fig.3 The nonlinear control system block diagram of the liquid level process based on $h$

Another nonlinear system control scheme shows in Fig.4. According to formula (6), we can get the flow resistance $R$ as

$R=\frac{\Delta h}{\Delta Q_{0}}$

Then the $K$ and $T$ can be calculated according to formula (10) and (11). So the basis of the nonlinear process parameter correction here is $\Delta h$ and $\Delta Q_{0}$. The corresponding block diagram is showed in Fig.5. This scheme needs an more flow transmitter.

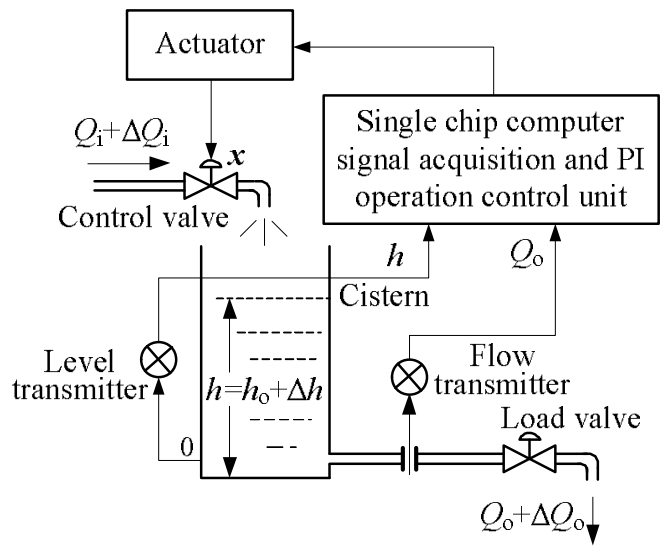

Fig.4 The nonlinear control system of the liquid level process based on $\Delta h$ and $\Delta Q_{\mathrm{o}}$

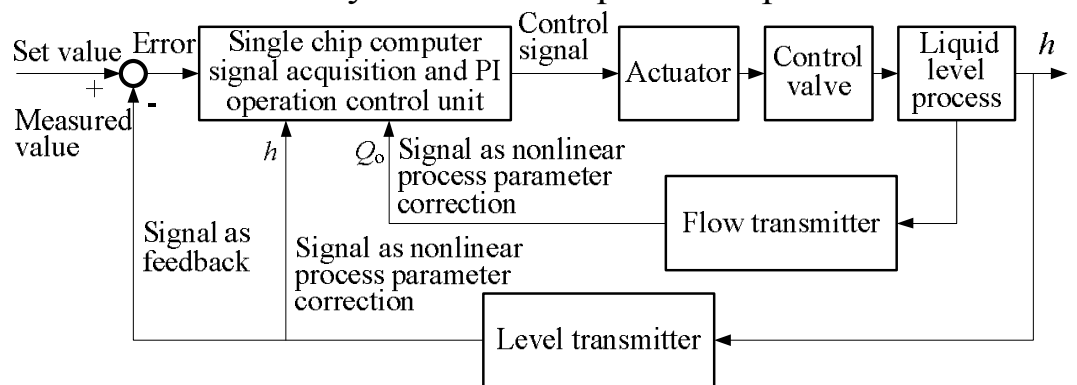

Fig.5 The nonlinear control system block diagram of the liquid level process based on $\Delta h$ and $\Delta Q_{\mathrm{o}}$

\section{The Test Results}

For a liquid level process experimental device, the diameter of cistern is $0.24 \mathrm{~m}$, the height is $0.35 \mathrm{~m}$, the load valve is in an opening, when the $h$ is change in $0.20 \sim 0.34 \mathrm{~m}$, through an experiment we can get the proportional coefficient $k$ of the load valve by formula (3), see table 1 . Let the $k$ is approximately equal to $0.014 \mathrm{~m}^{5 / 2} / \mathrm{s}$. Then the $R$ can be obtain by formula (13), it is change in $63.9 \sim 83.30 \mathrm{~s} / \mathrm{m}^{2}$. According to the control scheme shows in Fig.2 and Fig.3, using PI control strategy, but the integral time $T_{\mathrm{I}}$ not change and let the $T_{\mathrm{I}}=100 \mathrm{~s}$, the amplification coefficient $K_{\mathrm{c}}$ of the PI control unit is change with $R$ as follow

$K_{\mathrm{c}} \cdot K=k_{\mathrm{p}}$

or

$K_{\mathrm{c}}=\frac{k_{\mathrm{p}}}{K}=\frac{k_{\mathrm{p}}}{k_{\mathrm{x}} R}$

Where, $k_{\mathrm{p}} \longrightarrow$ the open loop amplification coefficient of the 4:1 attenuation process; 
$K_{\mathrm{c}} \longrightarrow$ the amplification coefficient of the PI control unit, $K_{\mathrm{c}}=1 / \mathrm{P}, \mathrm{P}$ is the proportioning of the PI control unit.

Table 1. The proportional coefficient $k$ of the load valve, $R$ and $\mathrm{P}$

\begin{tabular}{|c|c|c|c|c|}
\hline$h(\mathrm{~m})$ & $Q_{\mathrm{o}}\left(\mathrm{m}^{3} / \mathrm{s}\right)$ & $k\left(\mathrm{~m}^{5 / 2} / \mathrm{s}\right)$ & $R\left(\mathrm{~s} / \mathrm{m}^{2}\right)$ & $\mathrm{P}(\%)$ \\
\hline 0.20 & 0.006219 & 0.0139059 & 63.89 & 37.3 \\
\hline 0.25 & 0.006751 & 0.0135014 & 71.43 & 33.4 \\
\hline 0.30 & 0.007337 & 0.0133954 & 78.25 & 30.5 \\
\hline 0.35 & 0.007931 & 0.0134058 & 83.30 & 28.6 \\
\hline
\end{tabular}

Here $h$ is the basis of the nonlinear process parameter correction. When the $h$ is change in $0.20 \sim 0.34 \mathrm{~m}$, the proportioning $\mathrm{P}$ is change from 37.3 to $28.6 \%$. If run this liquid level process according to linear principles of automatic control the proportioning $\mathrm{P}$ is about $30 \%$. The test curves show in Fig.6. Curve 1 is linear system control strategy and curve 2 is nonlinear system control strategy. When the set value is step change from $0.22 \mathrm{~m}$ to $0.31 \mathrm{~m}$, the maximum overshoot is reduced by $16 \%$. It is obvious that nonlinear system control strategy is superior to linear system control strategy.

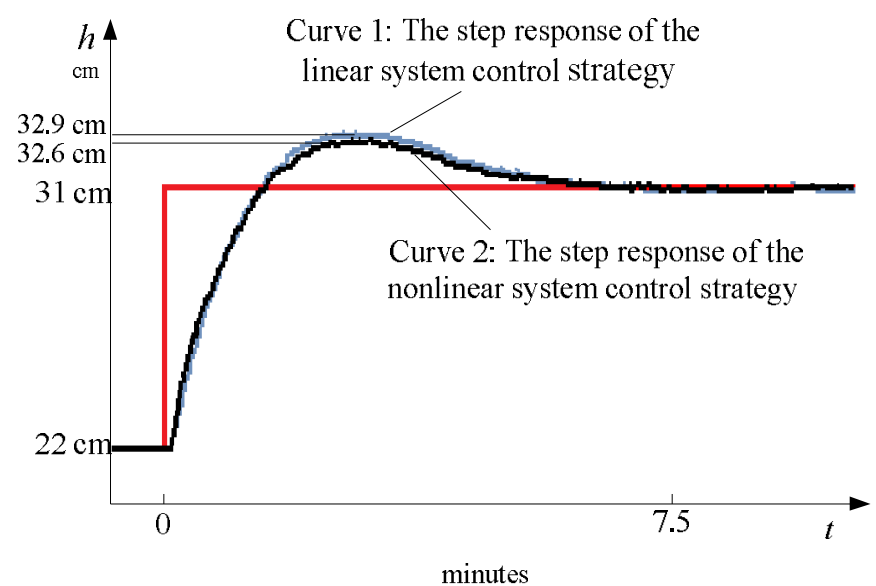

Fig.6 The step response curves of the linear and nonlinear system control strategy

\section{Conclusion}

For the liquid level process discussed above, so long as building the nonlinear system model, taking the nonlinear system control strategy, measuring the $h$ and $Q_{0}$ with higher precision, the nonlinear process parameters $K$ and $T$ under different $h$ can be calculated accurately. The parameter identification of the nonlinear process model is completed and the best control signal can be calculated out with the selected control algorithm. So the automatic control of nonlinear system can be realized and the better control effect can be achieved. The key of such nonlinear system control strategy is to find the basis of the nonlinear process parameter correction. For a few of industrial process we can get its model through theoretical analysis, and for most of the industrial process we can get the model through the experiment or experience only. As long as finding the basis of the nonlinear process parameter correction through the modeling we can realize nonlinear system automatic control easily with the single chip computer. This nonlinear system control strategy can be applied to industrial processes and expected to get better control effect then linear control strategy. The more serious nonlinear, the more obvious on the improvement of control effect.

\section{References}

[1] Ximmin Liu, (2015), Kong Zhi Yi Biao Ji Xi Tong, second edition, National Defense Industry Press, Beijing, Reference to chapter 6. In Chinese. 